\title{
An Empirical Evaluation of Cross-scene Crowd Counting Performance
}

\author{
Rita Delussu, Lorenzo Putzu, Giorgio Fumera \\ University of Cagliari, Piazza D'Armi, Cagliari, Italy \\ Department of Electrical and Electronic Engineering, Piazza D'armi, 09123 Cagliari, Italy \\ \{rita.delussu,lorenzo.putzu,fumera\}@unica.it
}

Keywords: Crowd counting, Crowd density estimation, Cross-scene evaluation, Video surveillance

\begin{abstract}
Crowd counting and density estimation are useful but also challenging tasks in many video surveillance systems, especially in cross-scene settings with dense crowds, if the target scene significantly differs from the ones used for training. This also holds for methods based on Convolutional Neural Networks (CNNs) which have recently boosted the performance of crowd counting systems, but nevertheless require massive amounts of annotated and representative training data. As a consequence, when training data is scarce or not representative of deployment scenarios, also CNNs may suffer from over-fitting to a different extent, and may hardly generalise to images coming from different scenes. In this work, we focus on real-world, challenging application scenarios when no annotated crowd images from a given target scene are available, and evaluate the cross-scene effectiveness of several regression-based state-of-the-art crowd counting methods, including CNN-based ones, through extensive cross-data set experiments. Our results show that some of the existing $\mathrm{CNN}$-based approaches are capable of generalising to target scenes which differ from the ones used for training in the background or lighting conditions, whereas their effectiveness considerably degrades under different perspective and scale.
\end{abstract}

\section{INTRODUCTION}

Automatic crowd counting and density estimation are useful functionalities in video surveillance applications. These tasks can be very challenging in unconstrained real-world scenarios, especially for dense crowd scenes with severe overlapping between people, perspective distortion and different lighting conditions. Several crowd counting and density estimation methods have been proposed so far (Loy et al., 2013; Sindagi and Patel, 2017a). Some of them are based on pedestrian detection or tracking, which are suitable only for sparse crowds with limited or no overlapping between people (Loy et al., 2013). Other methods, which are more suited to dense crowds, are based on regression techniques, either for crowd counting only (Loy et al., 2013) or also for density estimation (Sindagi and Patel, 2017a). The latter methods require a training set of crowd images manually annotated with the exact number of people.

In this work we focus on dense crowd scenarios, which are the ones we are addressing in the context of the LETSCROWD project funded by the European Commission under the $\mathrm{H} 2020$ programme related to the security of mass gathering events. ${ }^{1}$ In particular, we consider fully unsupervised cross-scene application scenarios where a system has to be deployed on a specific target scene for which it is not possible to collect and annotate images for training or fine-tuning, and additionally real-time operation is required. Although considerable progress has been achieved so far, especially by recent methods based on convolutional neural networks (CNNs) (Sindagi and Patel, 2017a), and some solutions based on domain adaptation or transfer learning have already been proposed, crowd counting and density estimation remain challenging tasks in a cross-scene setting like the one above. In particular, only limited cross-scene evaluations of existing methods have been provided in the respective papers. This is partly due also to the small number and relatively small size of publicly available data sets of dense crowds.

A thorough evaluation and analysis of the performance of existing methods under realistic crossscene settings is therefore still lacking in the literature. This is however a necessary step toward further development of crowd counting and density estimation methods that can be effectively deployed also

\footnotetext{
${ }^{1}$ https://letscrowd.eu/
} 
in challenging cross-scene application scenarios. Accordingly, the aim of this work is to evaluate the performance gap of state-of-the-art regression-based crowd counting methods between same-scene and cross-scene scenarios, i.e., where manually annotated images of the target scene are or are not available for training, respectively. To this aim we simulate crossscene settings using the available benchmark data sets, and consider several state-of-the-art regressionbased methods, including the most recent CNN-based ones, for which either a re-implementation was possible or the code was made available by the authors.

This paper is structured as follows. In Sect. 2 we review existing approaches and methods for crowd counting and density estimation. In Sect. 3 we discuss their open issues, focusing on the application scenario mentioned above, and describe the objective of this work. Our experimental evaluation is described in Sect. 4. The discussion of Sect. 4.4 concludes this paper and outlines directions for future work.

\section{RELATED WORK}

Several approaches have been developed so far for crowd counting and density estimation (Loy et al., 2013; Sindagi and Patel, 2017a). Existing methods can be categorised into counting by detection, counting by clustering, and counting by regression (Loy et al., 2013). The first two approaches are based on detecting or tracking each pedestrian in a scene, and can provide an exact count. However they are effective only on sparse crowd scenes with little or no overlapping among people (Loy et al., 2013). The latter one provides instead a direct mapping from low-level image features to the number of people (Loy et al., 2013) or, for most recent CNN-based methods, to the density map, from which the number of people can be derived (Sindagi and Patel, 2017a). This approach is suited to dense crowd scenes, but can provide only an estimate of the number of people and the corresponding density map. In the rest of this section, we focus on the regression-based approach.

\subsection{Early regression-based methods}

Early regression-based methods are based on extracting low-level image features (usually texture, gradient and edge), and on training a regression model to estimate the number of people in a given image. Some of them carry out also foreground segmentation (e.g., by background subtraction). Typical features are the grey-level co-occurrence matrix (GLCM) and Local Binary Patterns (LBP). Both linear and non-linear re- gression models have been proposed, such as partial least squares, kernel ridge regression, support vector regression with RBF kernel and Gaussian process regression (Loy et al., 2013).

\subsection{Methods based on CNNs}

More recent CNN-based methods estimate either the number of people or the density map. In the latter case the density map (ground truth) is obtained as the sum of Gaussian kernels centred on each pedestrian, whose position has to be manually annotated (Sindagi and Patel, 2017a). Existing methods are based either on specific CNN architectures, or on modifications of "standard" architectures such as VGG.

A specific Multi-Column CNN architecture (MCNN) was proposed in (Zhang et al., 2016), aimed at achieving robustness to scale variations. It is made up of three parallel CNNs with the same structure except for the dimensions of filters (large, medium and small), and a block used to merge the corresponding feature maps. Two similar architectures, Counting $\mathrm{CNN}(\mathrm{CCNN})$ and Hydra CNN, were proposed in (Onoro-Rubio and López-Sastre, 2016). CCNN computes the density maps of several image patches, and aggregates them to obtain the final density map. Hydra CNN improves the CCNN architecture by combining the information from multiple scales at the same time, inspired by other works (Marsden et al., 2017; Sindagi and Patel, 2017b). In particular, in (Sindagi and Patel, 2017b) a cascade CNN architecture (Cascade-CNN) was proposed to learn two related sub-tasks: crowd count classification and density map estimation. The first sub-task consists of categorising the crowd count into ten groups. The second one uses information extracted in the first sub-task to obtain the density map. The first layers are shared between the two sub-tasks. Despite the fact that all these methods have a high performance, their complexity might be high, and the training phase might take several hours. This issue was addressed in (Liu et al., 2018) and (Li et al., 2018). In (Liu et al., 2018) a scale-aware multi-task architecture fast to train was proposed. It extracts concentric image patches, and exploits the fact that inner patches contain a number of people lower or equal to larger ones. In the Congested Scene Recognition Network (CRSNet) of (Li et al., 2018) a dilated convolution which aggregates multi-scale information without increasing the number of parameters is used to keep processing time low. It consists of one front-end and four different backends: the front-end is based on VGG, in which the fully connected layers are replaced by convolutional layers; the back-end is composed by dilated convo- 
lutional layers to up-sample the feature maps to the original image resolution. The Deformation Aggregation network (DA-Net) of (Zou et al., 2018) computes the density map by aggregating the outputs of different layers. It is based on the VGG architecture to which new blocks are added to preserve the correspondence between the input image and the estimated density map, to improve robustness to scale variations. A soft-attention strategy is also used to dynamically weigh the feature maps of different layers. Similarly, in (Liu et al., 2019) an end-to-end architecture was proposed to fuse multi-scale contextual information (CAN) without using image patches. It is composed of the first ten layers of VGG-16 and a decoder based on dilated convolutions. The output of the first part of this architecture concatenates feature maps with weighted feature maps.

To limit cross-scene performance degradation, which is inherent in supervised methods, domain adaptation methods have been proposed. The Spatial Fully Connected Network (SFCN) model of (Wang et al., 2019) consists of a standard CNN architecture (VGG-16 or ResNet-101), a spatial encoder and a regression layer; it is trained on a synthetic data set obtained from a video game, and is fine tuned using real images from benchmark data sets. In (Sindagi and Patel, 2020) a Hierarchical Attention-based Crowd Counting Network was proposed: a spatial attention module selects relevant regions in the feature maps, and the global attention module produce a channelwise map. The network is fine-tuned on a data set with categorical, image-level density values (zero, very low, low density, etc.).

\section{OPEN ISSUES AND GOAL OF THIS WORK}

In this work we consider the following, very challenging real application scenario we are dealing with in the LETSCROWD project:

- a crowd counting system has to be deployed on a given target scene, which is different from the ones used for training, in terms of perspective, background, configuration of the people in the scene and possibly also crowd size;

- no annotated crowd images of the target scene can be collected for training or fine tuning the system;

- the system has to operate in real time.

Cross-scene effectiveness is a known issue of existing crowd counting and density estimation methods, but, to the best of our knowledge, it has been addressed explicitly only in (Zhang et al., 2015; Sindagi and Patel,
2020). The solution proposed in (Zhang et al., 2015) is based on the use of a perspective normalisation to compensate for perspective distortion. Using a perspective map is common in regression-based crowd counting methods (Loy et al., 2013); however it may be not sufficient if the target scene is significantly different from the ones used for training. The solution of (Sindagi and Patel, 2020) is partially supervised: it requires to collect representative images from the target scene, annotating them into three discrete density values (low, medium, high), and in using them to fine tune the proposed CNN architecture; this can be however infeasible in the application scenario considered in this work. Further solutions are fully unsupervised, but they still require the collection of representative images from the target scene (Liu et al., 2018; Sam et al., 2019). Additionally, several CNN-based solutions exhibit a high processing time in the inference step, which can prevent their use in real-time applications (Sindagi and Patel, 2017a). Some works reported a cross-scene evaluation of the proposed methods (Zhang et al., 2016), but limited to a single target data set. Moreover, some of these evaluations cannot be considered representative of the above application scenario where a crowd counting and density estimation system has to be deployed on a specific target scene, since benchmark data sets of dense crowds are made up of a collection of single crowd images taken from different scenes, with the only exception of World Expo Shanghai 2010 (see Sect. 4.2).

Evaluating the performance gap of existing crowd counting and density estimation methods between same-scene and cross-scene settings, and especially the cross-scene setting considered here, is therefore still an open issue and also a very relevant one. This is the goal of our ongoing work, inspired by our experience in the LETSCROWD project. In the rest of this paper we present the first results of an extensive empirical evaluation we are carrying out under the setting described above. To this aim we simulate the above cross-scene setting by using as the target scene a given data set of images from a single scene, and by using images from different scenes in the training set. Our evaluation is carried out on several state-of-theart regression-based methods, including CNN-based ones, for which either a re-implementation was possible or the code was made available by the authors.

\section{EXPERIMENTAL EVALUATION}

In this section we evaluate and compare the same- and cross- crowd counting performance of state-of-the-art methods on benchmark data sets. We first describe the 
methods and data sets we used, then the experimental set-up and finally the experimental results.

\subsection{Crowd counting methods}

We selected four representative regression-based methods (see Sect. 2.1): linear regression, Partial Least Squares (PLS) regression, Support Vector Regression (SVR) with Radial Basis Function (RBF) kernel, and Random Forest (RF) regression (Loy et al., 2013). Gaussian Process regression has been discarded since it turned out to be not suitable for real time applications. Most of the feature sets used by the above methods (see (Loy et al., 2013)) are significantly affected by the image background; even when a background image is available, existing background subtraction and segmentation approaches are not effective in real-world scenarios with frequent illumination changes. We decided therefore to use only the LBP texture descriptor. Moreover, here we have not used the perspective correction nor the region of interest mask (Loy et al., 2013; Ryan et al., 2015), to correct the distortion or the influence of the background. Indeed, this experiments should simulate a real case scenario (a new camera installation) where all these information and data are not available.

We also selected six more recent CNN-based methods (see Sect. 2.2) whose code was made available by the authors: MCNN, Cascade-CNN, DANet, CRSNet, CAN, and SFCN (see Sect. 2.2 for a description of these methods). We point out that for all the above CNN architectures, except for SFCN, we used the trained models provided by the same authors. For SFCN we trained the whole CNN instead, since no pre-trained model was available.

\subsection{Data sets}

To our knowledge, only three publicly available data sets can be considered representative of dense crowd scenarios (Zhang et al., 2019; Sindagi and Patel, 2017a), namely ShanghaiTech, UCF-QNRF and World Expo Shanghai 2010. However the first two do not contain images from a same scene, and World Expo Shanghai 2010 contains only 3 to 41 training images belonging to a same scene. They are therefore not suitable to be used to simulate target scenes in the testing sets of our cross-scene experiments, under the scenario of interest to this paper. We used only ShanghaiTech to train CNN-based models. To simulate the target scenes we used three other publicly available, single-scene data sets, namely Mall, UCSD and PETS. Although they do not include dense crowd images, they present challenging crowd scenes nevertheless, with lighting variations, perspective distortion and severe occlusions. Mall is made up of 2,000 frames from a single scene, collected from a surveillance camera in a shopping mall, with a size of $640 \times 480$. It contains a total of 62,325 pedestrians (Chen et al., 2012), with 13 to 53 people per frame (on average 31 ). This is a challenging data set with severe perspective distortion and several occlusions caused by static objects or by other people. Following the set-up used in recent works (Sindagi and Patel, 2017a) we used the first 800 frames as the training set and the remaining 1200 frames as the testing set. UCSD contains 2,000 frames of size $238 \times 158$ acquired from a camera installed in a pedestrian walkway at the UCSD campus (Chan et al., 2008). It contains a total of 49,885 pedestrians, with an average number of people per frame of around 25. Following the set-up of (Sindagi and Patel, 2017a) we split the data set into two parts: a training set containing frames from 600 to 1,399 , and a testing set containing the remaining frames. PETS2009 was released to test several algorithms for visual surveillance tasks (Ferryman and Shahrokni, 2009). The S1 part includes crowd counting: it is subdivided into three difficulty levels, defined by the crowdedness and behaviour of the people in the scene. Each level presents two sequences acquired at different times under different illumination and shading. This is not a standard singlescene data set since each sequence has been acquired with a different camera, but the frames belonging to the same camera view (for different difficulty levels and times) can be grouped to create single-scene data sets. To this aim we grouped the images from the first 3 cameras to create 3 single-scene data sets named PETSview1,PETSview2 and PETSview3; we used the ground truth provided in (Zhang and Chan, 2019). ShanghaiTech contains 1,198 images for a total of 330,165 pedestrians (Zhang et al., 2016). This data set is one of the most used in the literature, especially for training CNN models, since it includes images acquired from different cameras, with different illumination, perspective and crowd density. It is usually divided into two parts, Part_A and Part_B, containing 482 and 716 images, respectively. Each part is further subdivided into 300 images for training and the remaining ones for testing (Liu et al., 2018; Zhang et al., 2016; Sindagi and Patel, 2017a). shows one frame from the above data sets.

\subsection{Experimental set-up}

We consider two accuracy metrics commonly used for crowd counting methods, mean absolute error (MAE) 


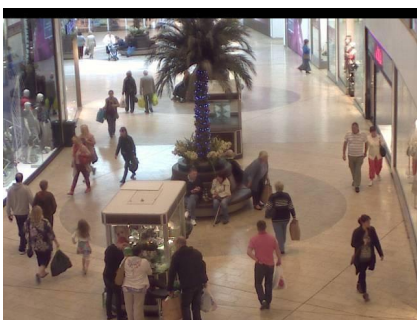

(a)

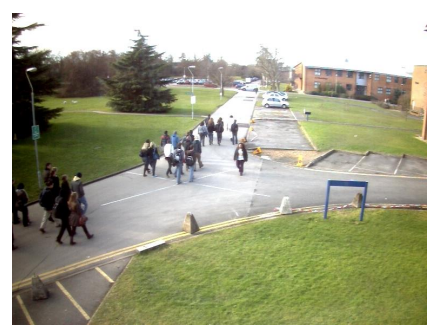

(d)

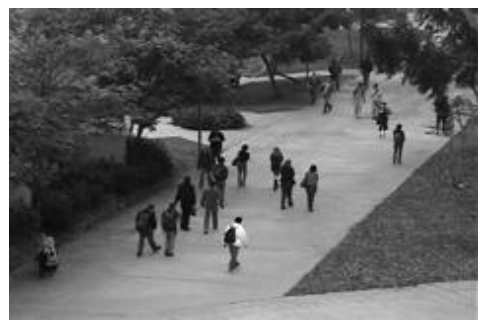

(b)

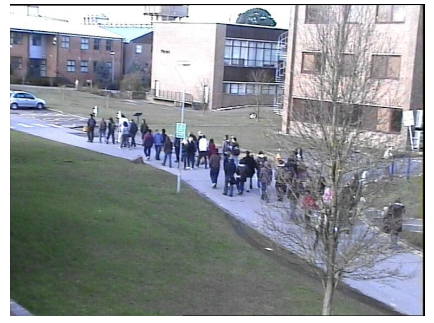

(e)

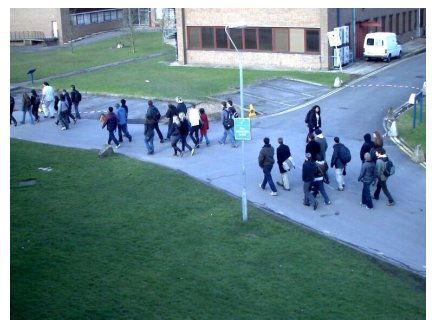

(c)

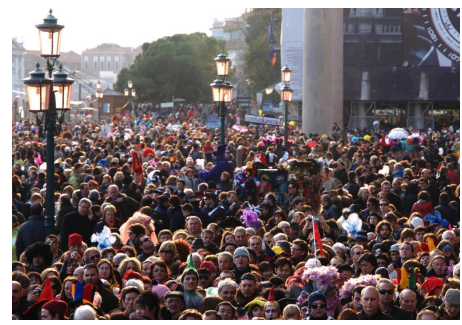

(f)

Figure 1: Example of frames from the data sets used in our experiments: (a) Mall, (b) UCSD, (c) PETSview1, (d) PETSview2, (e) PETSview3, (f) ShanghaiTech.

and mean root squared error (RMSE):

$$
\begin{aligned}
M A E & =\frac{1}{N} \sum_{i=1}^{N}\left|y_{i}-\hat{y}_{i}\right| \\
R M S E & =\left(\frac{1}{N} \sum_{i=1}^{N}\left(y_{i}-\hat{y}_{i}\right)^{2}\right)^{\frac{1}{2}}
\end{aligned}
$$

where $N$ is the number of images, $y_{i}$ is the exact people count in the $i$-th image (ground truth) and $\hat{y}_{i}$ the estimated count. Note that RMSE penalises large errors more heavily than small ones with respect to MAE. We point out that in many recent works (Liu et al., 2018; Wang et al., 2019; Ryan et al., 2015) the RMSE metric was used, but it was called MSE, although MSE does not include the square root (Loy et al., 2013). We use the RMSE to be aligned with the most recent works in this field, and also because it has the same unit of measurement as MAE.

In our experiments we simulated the cross-scene setting described at the beginning of Sect. 4.1 by using training images taken from one or several data sets, and testing the resulting model on images from a single scene taken from a different data set. As a baseline to evaluate the performance gap between samescene and cross-scene scenarios, for each model we also include the results obtained using training and testing samples from a same data set. Tables 1 and 2 show the results of the experiments carried out using training images taken from a single data set. For ease of comparison, for each target scene (data set) the same-scene performance is highlighted in grey.
To evaluate whether using as training data images coming from different scenes can improve crossscene performance, we also carried out experiments using as a training set the multi-scene data set ShangaiTech (either part_A or part_B). The results are reported in Table 3.

\subsection{Results}

From Table 1 it can be observed that in most cases the accuracy achieved in cross-scene experiments by CNN-based methods is definitely worse than that one achieved in the corresponding single-scene experiment (i.e., when training and testing images come from the same data set). The most noticeable performance gap can be observed when UCSD is used as the target scene (testing set). On the other hand, as it can be expected, when training images come from a different data set which however has a similar perspective and scale as the testing images (target scene) the cross-scene accuracy is comparable with the corresponding single-scene accuracy. This happens for the Mall and PETS data set, up to the point that in some cases the performance achieved on Mall is even better when the training set comes from PETS than from Mall itself. Similar behaviour can be observed when two of the three different views of the PETS data sets, which are very similar, are used in a cross-scene experiment. Instead, it is interesting to see from Table 2 that the cross-scene performances of methods not based on CNNs are mostly comparable to the ones of $\mathrm{CNN}$-based methods, whereas 
Table 1: Cross-scene MAE and RMSE of CNN-based methods. Same-scene accuracy (when training and testing come from the same data set) is reported for comparison, highlighted in grey. The best result for each column is reported in bold.

\begin{tabular}{|c|c|c|c|c|c|c|c|c|c|c|c|}
\hline \multirow{2}{*}{$\begin{array}{l}\vec{D} \\
\text { 总 } \\
\sum\end{array}$} & \multirow[t]{2}{*}{ Training set } & \multicolumn{2}{|c|}{ Mall } & \multicolumn{2}{|c|}{ UCSD } & \multicolumn{2}{|c|}{$\begin{array}{c}\text { Testing set } \\
\text { PETSview1 }\end{array}$} & \multicolumn{2}{|c|}{ PETSview2 } & \multicolumn{2}{|c|}{ PETSview3 } \\
\hline & & MAE & RMSE & MAE & RMSE & MAE & RMSE & MAE & RMSE & MAE & RMSE \\
\hline \multirow{5}{*}{ 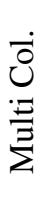 } & Mall & 5.33 & 6.17 & 24.64 & 25.75 & 5.94 & 7.83 & 9.67 & 10.95 & 9.9 & 11.22 \\
\hline & UCSD & 86.39 & 88.04 & 2.3 & 2.84 & 144.9 & 149.6 & 49.4 & 56.85 & 180.6 & 181.2 \\
\hline & PETSview1 & 19.54 & 20.16 & 24.18 & 25.28 & 6.2 & 7.86 & 22.05 & 23.59 & 9.77 & 11.75 \\
\hline & PETSview2 & 3.39 & 4.27 & 19.62 & 20.92 & 20.93 & 22.19 & 4.23 & 5.08 & 24.29 & 27.72 \\
\hline & PETSview3 & 4.31 & 5.35 & 21.28 & 22.47 & 19.54 & 21.63 & 10.37 & 11.66 & 4.18 & 5.13 \\
\hline \multirow{5}{*}{ 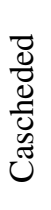 } & Mall & 5.53 & 6.39 & 23.42 & 24.58 & 5.77 & 7.42 & 17.65 & 19.28 & 11.41 & 12.79 \\
\hline & UCSD & 189.1 & 191.1 & 2.04 & 2.50 & 213.7 & 217.9 & 111.9 & 113.7 & 298.5 & 300.8 \\
\hline & PETSview1 & 9.93 & 10.73 & 24.18 & 25.13 & 5.11 & 6.29 & 15.56 & 17.20 & 4.46 & 5.95 \\
\hline & PETSview2 & 4.68 & 5.95 & 24.63 & 25.76 & 36.85 & 38.49 & 4.80 & 6.06 & 47.34 & 50.96 \\
\hline & PETSview3 & 4.61 & 5.79 & 21.94 & 23.12 & 21.90 & 24.54 & 11.50 & 13.97 & 4.23 & 5.06 \\
\hline \multirow{5}{*}{ 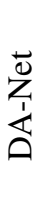 } & Mall & 5.43 & 6.42 & 25.42 & 26.54 & 7.51 & 9.43 & 11.7 & 13.14 & 8.84 & 10.27 \\
\hline & UCSD & 164.1 & 166.1 & 5.18 & 6.39 & 185.9 & 192.1 & 61.76 & 66.53 & 227.3 & 228.5 \\
\hline & PETSview1 & 7.97 & 9.06 & 26.1 & 27.09 & 4.92 & 6.15 & 16.41 & 19.12 & 6.34 & 7.74 \\
\hline & PETSview2 & 28.95 & 29.54 & 27.86 & 29.0 & 26.43 & 28.38 & 28.68 & 30.37 & 32.89 & 33.38 \\
\hline & PETSview3 & 7.9 & 9.48 & 18.8 & 20.12 & 18.02 & 20.45 & 13.2 & 15.15 & 4.63 & 5.92 \\
\hline \multirow{5}{*}{ 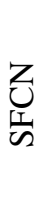 } & Mall & 4.05 & 5.02 & 28.15 & 29.27 & 19.37 & 20.85 & 27.66 & 28.72 & 71.38 & 71.87 \\
\hline & UCSD & 880.2 & 882.1 & 2.91 & 3.64 & 853.5 & 859.6 & 634.3 & 635.5 & 988.4 & 990.6 \\
\hline & PETSview1 & 8.33 & 9.64 & 27.13 & 28.1 & 6.32 & 7.57 & 12.83 & 14.5 & 10.74 & 12.05 \\
\hline & PETSview2 & 36.55 & 38.35 & 25.93 & 26.85 & 85.29 & 87.81 & 8.1 & 9.81 & 106.9 & 108.6 \\
\hline & PETSview3 & 14.78 & 15.98 & 28.23 & 29.36 & 11.49 & 13.64 & 10.03 & 12.74 & 4.35 & 5.68 \\
\hline \multirow{5}{*}{$\begin{array}{l}\overrightarrow{0} \\
\frac{\Delta}{\Delta} \\
\vec{v}\end{array}$} & Mall & 6.57 & 7.73 & 24.51 & 25.8 & 21.55 & 23.89 & 19.08 & 21.61 & 15.37 & 16.38 \\
\hline & UCSD & 70.78 & 71.46 & 6.2 & 7.01 & 57.52 & 61.86 & 28.29 & 31.21 & 69.06 & 69.36 \\
\hline & PETSview1 & 14.51 & 14.96 & 27.33 & 28.43 & 5.54 & 6.83 & 15.62 & 17.46 & 20.57 & 21.11 \\
\hline & PETSview2 & 12.15 & 12.66 & 27.06 & 28.16 & 10.14 & 11.82 & 7.09 & 7.9 & 8.42 & 9.53 \\
\hline & PETSview3 & 9.21 & 9.89 & 27.49 & 28.62 & 5.84 & 6.8 & 9.66 & 10.56 & 2.9 & 3.76 \\
\hline \multirow{5}{*}{$\underset{U}{Z}$} & Mall & 2.59 & 3.21 & 28.09 & 29.23 & 8.28 & 10.36 & 17.49 & 20.02 & 29.54 & 30.11 \\
\hline & UCSD & 281.6 & 283.1 & 4.73 & 6.16 & 173.5 & 176.9 & 133.4 & 135.2 & 252.0 & 252.4 \\
\hline & PETSview1 & 10.5 & 11.17 & 27.5 & 28.56 & 6.33 & 7.5 & 8.43 & 9.25 & 3.94 & 4.84 \\
\hline & PETSview2 & 27.59 & 28.51 & 27.1 & 28.15 & 24.62 & 26.03 & 6.07 & 7.67 & 5.09 & 6.77 \\
\hline & PETSview3 & 6.73 & 7.7 & 27.55 & 28.7 & 7.5 & 9.07 & 11.54 & 12.78 & 6.82 & 7.84 \\
\hline
\end{tabular}

(as it can be expected) the same-scene performance is generally better for CNN-based methods Table 1 .

Consider finally the cross-scene experiments on CNN-based methods where the multi-scene ShanghaiTech was used as the training set, whose results are reported in Table 3. First, the comparison with the results achieved in the same-scene setting (Table 1, grey entries) shows that in most cases the best crossscene performance on a given target scene (data set) is worse than the worst same-scene performance: this means that even using the multi-scene ShanghaiTech as the training set does not fill the gap with the samescene setting. If we compare the cross-scene performance in both tables, no consistent improvement can be observed when the training set comes from the multi-scene ShanghaiTech: in several cases (for the same CNN model) a better cross-scene performance is achieved when the training set comes from a singlescene data set.

\section{CONCLUSIONS}

We evaluated the gap between the same- and crossscene performance of several state-of-the-art crowd counting methods based on regression models and on CNNs, focusing on a challenging, real-world ap- 
Table 2: Cross-scene MAE and RMSE of regression models not based on CNNs. See caption of Table 1 for more details.

\begin{tabular}{|c|c|c|c|c|c|c|c|c|c|c|c|}
\hline \multirow{2}{*}{ 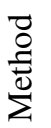 } & \multirow{2}{*}{ Training set } & \multicolumn{2}{|c|}{ Mall } & \multicolumn{2}{|c|}{ UCSD } & \multicolumn{2}{|c|}{$\begin{array}{l}\text { Testing set } \\
\text { PETSview1 }\end{array}$} & \multicolumn{2}{|c|}{ PETSview2 } & \multicolumn{2}{|c|}{ PETSview3 } \\
\hline & & MAE & RMSE & MAE & RMSE & MAE & RMSE & MAE & RMSE & MAE & RMSE \\
\hline \multirow{5}{*}{$\stackrel{\Xi}{\stackrel{\Xi}{\Xi}}$} & Mall & 2.74 & 3.49 & 9.59 & 11.63 & 289.2 & 294.4 & 348.7 & 349.0 & 268.1 & 270.9 \\
\hline & UCSD & 67.3 & 78.75 & 2.9 & 3.54 & 334.6 & 347.9 & 369.2 & 374.0 & 128.2 & 146.6 \\
\hline & PETSview1 & 276.9 & 277.0 & 577.1 & 577.2 & 6.25 & 7.91 & 33.43 & 38.04 & 9.35 & 11.17 \\
\hline & PETSview2 & 210.2 & 210.3 & 308.4 & 308.4 & 97.86 & 127.0 & 4.85 & 5.98 & 159.4 & 160.2 \\
\hline & PETSview3 & 12.15 & 14.01 & 29.09 & 29.93 & 110.3 & 110.7 & 125.1 & 126.6 & 6.84 & 8.42 \\
\hline \multirow{5}{*}{ 岌 } & Mall & 3.82 & 4.85 & 5.12 & 7.42 & 9.27 & 12.43 & 12.15 & 13.96 & 4.44 & 6.59 \\
\hline & UCSD & 5.83 & 6.98 & 3.82 & 4.66 & 9.12 & 11.45 & 8.06 & 10.46 & 5.22 & 5.94 \\
\hline & PETSview1 & 3.89 & 5.07 & 6.92 & 8.12 & 9.47 & 11.03 & 13.59 & 14.98 & 8.36 & 9.31 \\
\hline & PETSview2 & 6.88 & 8.57 & 5.38 & 7.31 & 8.01 & 8.94 & 9.56 & 11.05 & 6.27 & 8.14 \\
\hline & PETSview3 & 5.52 & 7.07 & 6.34 & 7.73 & 10.11 & 11.54 & 11.59 & 12.54 & 11.41 & 12.49 \\
\hline \multirow{5}{*}{$\frac{\vec{D}}{\vec{D}}$} & Mall & 4.8 & 6.29 & 8.15 & 9.18 & 9.56 & 10.45 & 9.8 & 10.68 & 8.74 & 9.55 \\
\hline & UCSD & 7.68 & 9.32 & 5.38 & 7.31 & 10.74 & 12.08 & 12.09 & 13.15 & 12.86 & 13.88 \\
\hline & PETSview1 & 12.26 & 13.57 & 6.21 & 8.52 & 12.82 & 15.25 & 14.85 & 16.79 & 17.67 & 18.56 \\
\hline & PETSview2 & 8.54 & 10.12 & 5.13 & 7.3 & 11.06 & 12.62 & 12.6 & 13.81 & 13.78 & 14.8 \\
\hline & PETSview3 & 5.11 & 6.71 & 7.52 & 8.61 & 9.76 & 10.61 & 10.2 & 11.04 & 9.5 & 10.37 \\
\hline \multirow{5}{*}{ 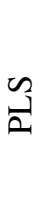 } & Mall & 3.16 & 4.1 & 110.7 & 110.9 & 51.97 & 65.77 & 16.97 & 20.94 & 53.4 & 61.05 \\
\hline & UCSD & 266.3 & 268.0 & 2.6 & 3.23 & 99.38 & 109.1 & 428.7 & 429.9 & 460.9 & 467.7 \\
\hline & PETSview1 & 49.0 & 49.37 & 13.0 & 14.21 & 8.46 & 10.13 & 20.39 & 24.53 & 21.07 & 26.56 \\
\hline & PETSview2 & 23.01 & 23.42 & 103.9 & 104.1 & 57.72 & 68.15 & 7.65 & 9.06 & 103.1 & 103.8 \\
\hline & PETSview3 & 18.05 & 18.67 & 5.1 & 7.27 & 14.55 & 16.86 & 25.12 & 26.75 & 9.03 & 10.06 \\
\hline
\end{tabular}

Table 3: Cross-scene MAE and RMSE of CNN-based methods trained on the multi-scene ShanghaiTech dataset. The best result for each column is reported in bold.

\begin{tabular}{|c|c|c|c|c|c|c|c|c|c|c|c|}
\hline & \multirow{2}{*}{$\begin{array}{c}\text { Training set } \\
\text { (ShanghaiTech) }\end{array}$} & \multicolumn{2}{|c|}{ Mall } & \multicolumn{2}{|c|}{ UCSD } & \multicolumn{2}{|c|}{$\begin{array}{l}\text { Testing set } \\
\text { PETSview } 1\end{array}$} & \multicolumn{2}{|c|}{ PETSview2 } & \multicolumn{2}{|c|}{ PETSview3 } \\
\hline & & MAE & RMSE & MAE & RMSE & MAE & RMSE & MAE & RMSE & MAE & RMSE \\
\hline \multirow{2}{*}{$\stackrel{U}{\Sigma}$} & Part A & 16.16 & 16.77 & 18.88 & 19.64 & 9.3 & 10.04 & 10.26 & 11.98 & 33.9 & 38.67 \\
\hline & Part B & 21.03 & 21.58 & 22.01 & 22.86 & 7.51 & 8.58 & 23.2 & 24.86 & 6.55 & 8.12 \\
\hline \multirow{2}{*}{$\dot{\tilde{U}}$} & Part A & 17.71 & 18.33 & 21.0 & 21.84 & 8.51 & 9.39 & 10.36 & 11.92 & 33.46 & 40.68 \\
\hline & Part B & 13.92 & 14.6 & 22.26 & 23.02 & 10.32 & 11.38 & 17.95 & 19.89 & 9.61 & 12.39 \\
\hline \multirow{2}{*}{ Z } & Part A & 16.76 & 17.32 & 23.96 & 24.67 & 8.88 & 10.21 & 14.49 & 16.56 & 15.68 & 16.68 \\
\hline & Part B & 18.02 & 18.64 & 22.82 & 24.01 & 8.93 & 10.71 & 19.19 & 22.03 & 20.13 & 21.11 \\
\hline \multirow{2}{*}{$\begin{array}{l}Z \\
\text { Z } \\
\text { U }\end{array}$} & Part A & 773.2 & 777.4 & 5.42 & 7.55 & 30.59 & 31.5 & 802.1 & 802.3 & 683.6 & 687.4 \\
\hline & Part B & 31.21 & 32.4 & 322.7 & 323.7 & 10.88 & 12.46 & 238.5 & 238.5 & 33.8 & 34.3 \\
\hline \multirow{2}{*}{$\frac{\alpha}{u}$} & Part A & 14.64 & 15.1 & 26.58 & 27.63 & 8.58 & 10.08 & 8.92 & 10.17 & 15.45 & 16.55 \\
\hline & Part B & 10.61 & 11.1 & 28.06 & 29.2 & 10.97 & 12.11 & 12.28 & 13.83 & 15.44 & 16.62 \\
\hline \multirow{2}{*}{ Z } & Part A & 9.72 & 10.28 & 27.04 & 28.16 & 5.04 & 5.87 & 6.2 & 7.46 & 10.3 & 11.67 \\
\hline & Part B & 3.6 & 4.56 & 28.05 & 29.18 & 6.53 & 8.25 & 10.31 & 11.49 & 15.57 & 16.55 \\
\hline
\end{tabular}

plication scenario where no manually annotated images of a specific target scene are available. To this aim we simulated cross-scene scenarios by training each model on one or more data sets, and then testing it on a different, single-scene data set. Our results show that some of the existing $\mathrm{CNN}$ models can 
achieve a relatively good performance also in crossscene scenarios, and that this happens when the target scene differs from the ones used for training only in the background or in the lighting conditions, but exhibit similar perspective and scale; their performance is considerably worse when target and training scenes significantly differ in perspective and scale, instead. As a possible solution to improve crossscene effectiveness when no manually annotated data from the target scene is available, and it is also difficult to obtain non-annotated data for unsupervised domain adaptation methods, we envisage the use of synthetic data sets reproducing the same perspective of the target scene. We are currently investigating this approach, and preliminary results can be found in (Delussu et al., 2020).

As a final remark, the still large gap between same- and cross-scene performance suggests to avoid focusing future work on improving crowd counting accuracy on benchmark data sets under same-scene scenarios (somewhat according to the suggestions given in (Torralba et al., 2011) for other computer vision tasks), and to address the efforts toward achieving a higher invariance in perspective and scale.

\section{ACKNOWLEDGEMENT}

This work was supported by the project "Law Enforcement agencies human factor methods and Toolkit for the Security and protection of CROWDs in mass gatherings" (LETSCROWD), EU Horizon 2020 programme, grant agreement No. 740466.

\section{REFERENCES}

Chan, A. B., Liang, Z.-S. J., and Vasconcelos, N. (2008). Privacy preserving crowd monitoring: Counting people without people models or tracking. In IEEE CVPR, pages 1-7.

Chen, K., Loy, C. C., Gong, S., and Xiang, T. (2012). Feature mining for localised crowd counting. In $B M V C$, pages $1-11$.

Delussu, R., Putzu, L., and Fumera, G. (2020). Investigating synthetic data sets for crowd density estimation. In VISAPP. In press.

Ferryman, J. and Shahrokni, A. (2009). Pets2009: Dataset and challenge. In PETS, pages 1-6.

Li, Y., Zhang, X., and Chen, D. (2018). Csrnet: Dilated convolutional neural networks for understanding the highly congested scenes. In IEEE CVPR, pages 10911100 .

Liu, W., Salzmann, M., and Fua, P. (2019). Context-aware crowd counting. In IEEE CVPR, pages 5099-5108.
Liu, X., van de Weijer, J., and Bagdanov, A. D. (2018). Leveraging unlabeled data for crowd counting by learning to rank. In IEEE CVPR.

Loy, C. C., Chen, K., Gong, S., and Xiang, T. (2013). Crowd counting and profiling: Methodology and evaluation. In Modeling, simulation and visual analysis of crowds, pages 347-382. Springer.

Marsden, M., McGuinness, K., Little, S., and O'Connor, N. E. (2017). Resnetcrowd: A residual deep learning architecture for crowd counting, violent behaviour detection and crowd density level classification. In IEEE AVSS, pages 1-7.

Onoro-Rubio, D. and López-Sastre, R. J. (2016). Towards perspective-free object counting with deep learning. In $E C C V$, pages 615-629. Springer.

Ryan, D., Denman, S., Sridharan, S., and Fookes, C. (2015). An evaluation of crowd counting methods, features and regression models. Computer Vision and Image Understanding, 130:1-17.

Sam, D. B., Sajjan, N. N., Maurya, H., and Babu, R. V. (2019). Almost unsupervised learning for dense crowd counting. In Association for the Advancement of Artificial Intelligence.

Sindagi, V. and Patel, V. M. (2017a). A survey of recent advances in cnn-based single image crowd counting and density estimation. Pattern Recognition Letters, 107:3-16.

Sindagi, V. A. and Patel, V. M. (2017b). Cnn-based cascaded multi-task learning of high-level prior and density estimation for crowd counting. In IEEE AVSS, pages $1-6$.

Sindagi, V. A. and Patel, V. M. (2020). HA-CCN: hierarchical attention-based crowd counting network. IEEE Trans. on Image Processing, 29:323-335.

Torralba, A., Efros, A. A., et al. (2011). Unbiased look at dataset bias. In IEEE CVPR, pages 1521-1528.

Wang, Q., Gao, J., Lin, W., and Yuan, Y. (2019). Learning from synthetic data for crowd counting in the wild. In IEEE CVPR, pages 8198-8207.

Zhang, C., Li, H., Wang, X., and Yang, X. (2015). Crossscene crowd counting via deep convolutional neural networks. In IEEE CVPR, pages 833-841.

Zhang, Q. and Chan, A. B. (2019). Wide-area crowd counting via ground-plane density maps and multi-view fusion cnns. In IEEE CVPR, page 82978306.

Zhang, Y., Zhou, C., Chang, F., and Kot, A. C. (2019). A scale adaptive network for crowd counting. Neurocomputing, 362:139-146.

Zhang, Y., Zhou, D., Chen, S., Gao, S., and Ma, Y. (2016). Single-image crowd counting via multi-column convolutional neural network. In IEEE CVPR, pages 589597.

Zou, Z., Su, X., Qu, X., and Zhou, P. (2018). Da-net: Learning the fine-grained density distribution with deformation aggregation network. IEEE Access, 6:6074560756. 